\title{
On Robust Hybrid Force/Motion Control Strategies Based on Actuator Dynamics for Nonholonomic Mobile Manipulators
}

\author{
Yongxin Zhu' ${ }^{1}$ and Liping Fan ${ }^{1,2}$ \\ ${ }^{1}$ School of Microelectronics, Shanghai Jiao Tong University, Shanghai 200240, China \\ ${ }^{2}$ School of Medical Instrument and Food Engineering, University of Shanghai for Science and Technology, \\ Shanghai 200093, China \\ Correspondence should be addressed to Liping Fan, fanlipingsds@gmail.com
}

Received 1 March 2012; Accepted 25 May 2012

Academic Editor: Weihai Zhang

Copyright (C) 2012 Y. Zhu and L. Fan. This is an open access article distributed under the Creative Commons Attribution License, which permits unrestricted use, distribution, and reproduction in any medium, provided the original work is properly cited.

\begin{abstract}
Robust force/motion control strategies are presented for mobile manipulators under both holonomic and nonholonomic constraints in the presence of uncertainties and disturbances. The controls are based on structural knowledge of the dynamics of the robot, and the actuator dynamics is also taken into account. The proposed control is robust not only to structured uncertainty such as mass variation but also to unstructured one such as disturbances. The system stability and the boundness of tracking errors are proved using Lyapunov stability theory. The proposed control strategies guarantee that the system motion converges to the desired manifold with prescribed performance. Simulation results validate that not only the states of the system asymptotically converge to the desired trajectory, but also the constraint force asymptotically converges to the desired force.
\end{abstract}

\section{Introduction}

Mobile manipulators refer to robotic manipulators mounted on mobile platforms. Such systems combine the advantages of mobile platforms and robotic arms and reduce their drawbacks [1-4]. For instance, the mobile platform extends the arm workspace, whereas the arm offers much operational functionality. Applications for such systems could be found in mining, construction, forestry, planetary exploration, teleoperation, and military [5-11].

Mobile manipulators possess complex and strongly coupled dynamics of mobile platforms and manipulators [12-16]. A control approach by nonlinear feedback linearization was presented for the mobile platform so that the manipulator is always positioned at the preferred configurations measured by its manipulability [17]. In [14], the effect of the dynamic interaction on the tracking performance of a mobile manipulator was studied, and 
nonlinear feedback control for the mobile manipulator was developed to compensate the dynamic interaction. In [18], a basic framework for the coordination and control of vehiclearm systems was presented, which consists of two basic task-oriented control: end-effector task control and platform self-posture control. The standard definition of manipulability was generalized to the case of mobile manipulators, and the optimization of criteria inherited from manipulability considerations were given to generate the controls of the system when its end-effector motion was imposed [19]. In [20], a unified model for mobile manipulator was derived, and nonlinear feedback was applied to linearize and decouple the model, and decoupled force/position control of the end-effector along the same direction for mobile manipulators was proposed and applied to nonholonomic cart pushing. The previously mentioned literature concerning with control of the mobile manipulator requires the precise information on the dynamics of the mobile manipulator; there may be some difficulty in implementing them on the real system in practical applications.

Different researchers have investigated adaptive controls to deal with dynamics uncertainty of mobile manipulators. Adaptive neural-network- (NN-) based controls for the arm and the base had been proposed for the motion control of a mobile manipulator [21, 22]; each NN control output comprises a linear control term and a compensation term for parameter uncertainty and disturbances. Adaptive control was proposed for trajectory/force control of mobile manipulators subjected to holonomic and nonholonomic constraints with unknown inertia parameters $[23,24]$, which ensures the state of the system to asymptotically converge to the desired trajectory and force. The principal limitation associated with these schemes is that controllers are designed at the velocity input level or torque input level, and the actuator dynamics are excluded.

As demonstrated in [25-27], actuator dynamics constitute an important component of the complete robot dynamics, especially in the case of high-velocity movement and highly varying loads. Many control methods have therefore been developed to take into account the effects of actuator dynamics (see, e.g., [28-30]). However, the literature is sparse on the control of the nonholonomic mobile manipulators including the actuator dynamics. In most of the research works for controlling mobile manipulators, joint torques are control inputs though in reality joints are driven by actuators (e.g., DC motors), and therefore using actuator input voltages as control inputs is more realistic. To this effect, actuator dynamics is combined with the mobile manipulator's dynamics in this paper.

This paper addresses the problem of stabilization of force/motion control for a class of mobile manipulator systems with both holonomic and nonholonomic constraints in the parameter uncertainties and external disturbances.

Unlike the force/motion control presented in [31-37], which is proposed for the mechanical systems subject to either holonomic or nonholonomic constraints, in our paper, the control is to deal with the system subject to both holonomic and nonholonomic constraints. After the dynamics based on decoupling force/motion is first presented, the robust motion/force control is proposed for the system under the consideration of the actuator dynamics uncertainty to complete the trajectory / force tracking. The paper has main contributions listed as follows.

(i) Decoupling robust motion/force control strategies are presented for mobile manipulator with both holonomic and nonholonomic constraints in the parameter uncertainties and external disturbances, and nonregressor-based control design is developed in a unified manner without imposing any restriction on the system dynamics. 
(ii) The actuators (e.g., DC motor) dynamics of both the mobile platform and the arm are integrated with mobile manipulator dynamics and kinematics so that the actuator input voltages are the control inputs thus making the system more realistic. control law.

Simulation results are described in detail that show the effectiveness of the proposed

The rest of the paper is organized as follows. The system description of mobile manipulator subject to nonholonomic constraints and holonomic is briefly described in Section 2. Problem statement for the system control is given in Section 4 . The main results of robust adaptive control design are presented in Section 5. Simulation studies are presented by comparison between the proposed robust control with nonrobust control in Section 6. Concluding remarks are given in Section 7.

\section{System Description}

Consider an $n$ DOF mobile manipulator with nonholonomic mobile base. The constrained mechanical system can be described as

$$
M(q) \ddot{q}+C(q, \dot{q}) \dot{q}+G(q)+d(t)=B(q) \tau+f
$$

where $q=\left[q_{1}, \ldots, q_{n}\right]^{T} \in R^{n}$ denote the generalized coordinates; $M(q) \in R^{n \times n}$ is the symmetric bounded positive definite inertia matrix; $C(\dot{q}, q) \dot{q} \in R^{n}$ denotes the Centripetal and Coriolis torques; $G(q) \in R^{n}$ is the gravitational torque vector; $d(t)$ denotes the external disturbances; $\tau \in R^{m}$ is the control inputs; $B(q) \in R^{n \times m}$ is a full rank input transformation matrix and is assumed to be known because it is a function of fixed geometry of the system; $f \in R^{m}$ denotes the vector of constraint forces; $J \in R^{n \times m}$ is Jacobian matrix; $\lambda=\left[\lambda_{n}, \lambda_{h}\right] \in R^{m}$ is Lagrange multipliers corresponding to the nonholonomic and holonomic constraints.

The generalized coordinates may be separated into two sets $q=\left[q_{v}, q_{a}\right]^{T}$, where $q_{v} \in$ $R^{v}$ describes the generalized coordinates for the mobile platform, $q_{a} \in R^{r}$ is the coordinates of the manipulator, and $n=v+r$.

Assumption 2.1 (see [38-40]). The mobile manipulator is subject to known nonholonomic constraints.

Assumption 2.2. The system (2.8) is subjected to $k$ independent holonomic constraints, which can be written as

$$
h(q)=0, \quad h(q) \in R^{k}
$$

where $h(q)$ is full rank, then $J(q)=\partial h / \partial q$.

Remark 2.3. In actual implementation, we can adopt the methods of producing enough friction between the wheels of the mobile platform and the ground such that this assumption holds [41-43]. 
The vehicle is subjected to nonholonomic constraints, the $l$ nonintegrable and independent velocity constraints can be expressed as

$$
A\left(q_{v}\right) \dot{q}_{v}=0
$$

where $A\left(q_{v}\right)=\left[A_{1}^{T}\left(q_{v}\right), \ldots, A_{l}^{T}\left(q_{v}\right)\right]^{T}: R^{v} \rightarrow R^{l \times v}$ is the kinematic constraint matrix which is assumed to have full rank $l$. In the paper, the vehicle is assumed to be completely nonholonomic. The effect of the constraints can be viewed as a restriction of the dynamics on the manifold $\Omega_{n}$ as

$$
\Omega_{n}=\left\{\left(q_{v}, \dot{q}_{v}\right) \mid A\left(q_{v}\right) \dot{q}_{v}=0\right\}
$$

The generalized constraint forces for the nonholonomic constraints can be given by

$$
f_{n}=A^{T}\left(q_{v}\right) \lambda_{n}
$$

Assume that the annihilator of the codistribution spanned by the covector fields $A_{1}\left(q_{v}\right), \ldots, A_{l}\left(q_{v}\right)$ is a $(v-l)$-dimensional smooth nonsingular distribution $\Delta$ on $R^{v}$. This distribution $\Delta$ is spanned by a set of $(v-l)$ smooth and linearly independent vector fields $H_{1}\left(q_{v}\right), \ldots, H_{v-l}\left(q_{v}\right)$; that is, $\Delta=\operatorname{span}\left\{H_{1}\left(q_{v}\right), \ldots, H_{v-l}(q)\right\}$, which satisfy, in local coordinates, the following relation:

$$
H^{T}\left(q_{v}\right) A^{T}\left(q_{v}\right)=0
$$

where $H\left(q_{v}\right)=\left[H_{1}\left(q_{v}\right), \ldots, H_{n_{v}-l}\left(q_{v}\right)\right] \in R^{v \times(v-l)}$. Note that $H^{T} H$ is of full rank. Constraints (2.3) imply the existence of vector $\dot{\eta} \in R^{v-l}[44]$, such that

$$
\dot{q}_{v}=H\left(q_{v}\right) \dot{\eta}
$$

Considering the nonholonomic constraints (2.3) and its derivative, the dynamics of mobile manipulator can be expressed as

$$
\begin{aligned}
& {\left[\begin{array}{cc}
H^{T} M_{v} H & H^{T} M_{v a} \\
M_{a v} H & M_{a}
\end{array}\right]\left[\begin{array}{c}
\ddot{\eta} \\
\ddot{q}_{a}
\end{array}\right]+\left[\begin{array}{cc}
H^{T} M_{v} \dot{H}+H^{T} C_{v} H & H^{T} C_{v a} \\
M_{a v} \dot{H}+C_{a v} H & C_{a}
\end{array}\right]\left[\begin{array}{c}
\dot{\eta} \\
\dot{q}_{a}
\end{array}\right]+\left[\begin{array}{c}
H^{T} G_{v} \\
G_{a}
\end{array}\right]+\left[\begin{array}{c}
H^{T} d_{v} \\
d_{a}
\end{array}\right]} \\
& \quad=\left[\begin{array}{c}
H^{T} B_{v} \tau_{v} \\
B_{\mathrm{a}} \tau_{a}
\end{array}\right]+\left[\begin{array}{cc}
0 & 0 \\
J_{v} & J_{a}
\end{array}\right]^{T}\left[\begin{array}{c}
0 \\
\lambda_{h}
\end{array}\right] .
\end{aligned}
$$

From Assumption 2.2, the holonomic constraint force $f_{h}$ can be converted to the joint space as $f_{h}=J^{T} \lambda_{h}$. Hence, the holonomic constraint on the robot's end effector can be viewed as restricting only the dynamics on the constraint manifold $\Omega_{h}$ defined by $\Omega_{h}=$ $\{(q, \dot{q}) \mid h(q)=0, J(q) \dot{q}=0\}$. The vector $q_{a}$ can be further rearranged and partitioned 
into $q_{a}=\left[q_{a}^{1}, q_{a}^{2}\right]^{T} ; q_{a}^{1} \in R^{r-k}$ describes the constrained motion of the manipulator, and $q_{a}^{2} \in R^{k}$ denotes the remaining joint variable. Then,

$$
J(q)=\left[\frac{\partial h}{\partial \eta}, \frac{\partial h}{\partial q_{a}^{1}}, \frac{\partial h}{\partial q_{a}^{2}}\right]
$$

From [45], it could be concluded $q$ is the function of $\zeta=\left[\eta, q_{a}^{1}\right]^{T}$, that is, $q=q(\zeta)$, and we have $\dot{q}=L(\zeta) \dot{\xi}$, where $L(\zeta)=\partial q / \partial \zeta, \ddot{q}=L(\zeta) \ddot{\zeta}+\dot{L}(\zeta) \dot{\zeta}$, and $L(\zeta), J^{1}(\zeta)=J(q(\zeta))$ satisfy the relationship

$$
L^{T}(\zeta) J^{1 T}(\zeta)=0
$$

The dynamic model (2.8), when it restricted to the constraint surface, can be transformed into the reduced model:

$$
M^{1} L(\zeta) \ddot{\zeta}+C^{1} \dot{\zeta}+G^{1}+d^{1}(t)=u+J^{1 T} \lambda_{h}
$$

where

$$
\begin{gathered}
M^{1}=\left[\begin{array}{cc}
H^{T} M_{v} H & H^{T} M_{v a} \\
M_{a v} H & M_{a}
\end{array}\right], \\
C^{1}=\left[\begin{array}{cc}
H^{T} M_{v} \dot{H} & H^{T} M_{v a} \\
M_{a v} H & M_{a}
\end{array}\right] \dot{L}(\zeta)+\left[\begin{array}{cc}
H^{T} M_{v} \dot{H}+H^{T} C_{v} H & H^{T} C_{v a} \\
M_{a v} \dot{H}+C_{a v} & C_{a}
\end{array}\right] L(\zeta), \\
G^{1}=\left[\begin{array}{c}
H^{T} G_{v} \\
G_{a}
\end{array}\right], \quad d^{1}(t)=\left[\begin{array}{c}
H^{T} d_{v} \\
d_{a}
\end{array}\right], \\
u=B^{1} \tau, \quad B^{1}=\left[\begin{array}{cc}
H^{T} B_{v} & 0 \\
0 & B_{a}
\end{array}\right], \quad \zeta=\left[\begin{array}{c}
\eta \\
q_{a}^{1}
\end{array}\right] .
\end{gathered}
$$

Multiplying $L^{T}$ by both sides of (2.11), we can obtain

$$
M_{L}(\zeta) \ddot{\zeta}+C_{L}(\zeta, \dot{\zeta}) \dot{\zeta}+G_{L}+d_{L}(t)=L^{T} B^{1} \tau
$$

The force multipliers $\lambda_{h}$ can be obtained by (2.11):

$$
\lambda_{h}=Z(\zeta)\left(C^{1}(\zeta, \dot{\zeta}) \dot{\zeta}+G^{1}+d^{1}(t)-B^{1} \tau\right)
$$

where $M_{L}=L^{T} M^{1} L, C_{L}=L^{T} C^{1}, G_{L}=L^{T} G^{1}, Z=\left(J^{1}\left(M^{1}\right)^{-1} J^{1 T}\right)^{-1} J^{1}\left(M^{1}\right)^{-1}$.

Property 1. The matrix $M_{L}$ is symmetric and positive definite.

Property 2. The matrix $\dot{M}_{L}-2 C_{L}$ is skew symmetric. 
Property 3 (see [46]). For holonomic systems, matrices $J^{1}(\zeta), L(\zeta)$ are uniformly bounded and uniformly continuous if $\zeta$ is uniformly bounded and continuous, respectively.

Property 4. There exist some finite positive constants $c_{i}>0(1 \leq i \leq 4)$ and finite nonnegative constant $c_{i} \geq 0(i=5)$ such that for all $\zeta \in R^{n}$, for all $\xi \in R^{n},\left\|M_{L}(\zeta)\right\| \leq c_{1},\left\|C_{L}(\zeta, \zeta)\right\| \leq$ $c_{2}+c_{3}\|\dot{\zeta}\|,\left\|G_{L}(\zeta)\right\| \leq c_{4}$, and $\sup _{t \geq 0}\left\|d_{L}(t)\right\| \leq c_{5}$.

\section{Actuator Dynamics}

The joints of the mobile manipulators are assumed to be driven by DC motors. Consider the following notations used to model a DC motor: $v \in R^{m}$ represents the control input voltage vector; $I$ denotes an $m$-element vector of motor armature current; $K_{N} \in R^{m \times m}$ is a positive definite diagonal matrix which characterizes the electromechanical conversion between current and torque; $L_{a}=\operatorname{diag}\left[L_{a 1}, L_{a 2}, L_{a 3}, \ldots, L_{a m}\right], R_{a}=\operatorname{diag}\left[R_{a 1}, R_{a 2}, R_{a 3}, \ldots, R_{a m}\right], K_{e}=$ $\operatorname{diag}\left[K_{e 1}, K_{e 2}, K_{e 3}, \ldots, K_{e m}\right], \omega=\left[\omega_{1}, \omega_{2}, \ldots, \omega_{m}\right]^{T}$ represent the equivalent armature inductances, resistances, back EMF constants, angular velocities of the driving motors, respectively; $G_{r}=\operatorname{diag}\left(g_{r i}\right) \in R^{m \times m}$ denotes the gear ratio for $m$ joints; $\tau_{m}$ are the torque exerted by the motor. In order to apply the DC servomotors for actuating an $n$-DOF mobile manipulator, assuming no energy losses, a relationship between the $i$ th joint velocity $\dot{q}_{i}$ and the motor shaft velocity $\omega_{i}$ can be presented as $g_{r i}=\omega_{i} / \dot{q}_{i}=\tau_{i} / \tau_{m i}$ with the gear ratio of the $i$ th joint $g_{r i}$, the $i$ th motor shaft torque $\tau_{m i}$, and the $i$ th joint torque $\tau_{i}$. The motor shaft torque is proportional to the motor current $\tau_{m}=K_{N} I$. The back EMF is proportional to the angular velocity of the motor shaft; then we can obtain

$$
L_{a} \frac{d I}{d t}+R_{a} I+K_{e} \omega=v
$$

In the actuator dynamics (3.1), the relationship between $\omega$ and $\dot{\zeta}$ is dependent on the type of mechanical system and can be generally expressed as

$$
\omega=G_{r} T \dot{\zeta} .
$$

The structure of $T$ depends on the mechanical systems to be controlled. For instance, in the simulation example, a two-wheel differential drive 2-DOF mobile manipulator is used to illustrate the control design. From [47], we have

$$
\begin{gathered}
v=\frac{\left(r \dot{\theta}_{l}+r \dot{\theta}_{r}\right)}{2}, \\
\dot{\theta}=\frac{\left(r \dot{\theta}_{r}-r \dot{\theta}_{l}\right)}{2 l}, \\
\dot{\theta}_{1}=\dot{\theta}_{1}, \\
\dot{\theta}_{2}=\dot{\theta}_{2},
\end{gathered}
$$




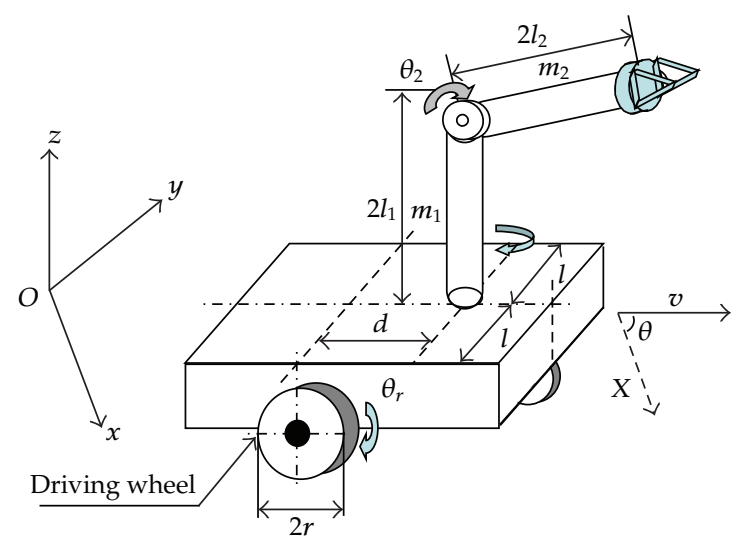

Figure 1: The 2-DOF mobile manipulator.

where $\dot{\theta}_{l}$ and $\dot{\theta}_{r}$ are the angular velocities of the two wheels, respectively, and $v$ is the linear velocity of the mobile platform, as shown in Figure 1 . Since $\dot{y}=v \cos \theta$, we have

$$
\begin{aligned}
& {\left[\begin{array}{llll}
\dot{\theta}_{l} & \dot{\theta}_{r} & \dot{\theta}_{1} & \dot{\theta}_{2}
\end{array}\right]^{T}=T\left[\begin{array}{llll}
\dot{y} & \dot{\theta} & \dot{\theta}_{1} & \dot{\theta}_{2}
\end{array}\right]^{T} \text {, }} \\
& T=\left[\begin{array}{cccc}
\frac{1}{r \cos \theta} & \frac{l}{r} & 0 & 0 \\
\frac{1}{r \cos \theta} & -\frac{l}{r} & 0 & 0 \\
0 & 0 & 1 & 0 \\
0 & 0 & 0 & 1
\end{array}\right],
\end{aligned}
$$

where $r$ and $l$ are shown in Figure 1.

Eliminating $\omega$ from the actuator dynamics (3.1) by substituting (3.2), one obtains

$$
\begin{gathered}
L^{T} B^{1} G_{r} K_{N} I=M_{L}(\zeta) \ddot{\zeta}+C_{L}(\zeta, \dot{\zeta}) \dot{\zeta}+G_{L}+d_{L}(t) \\
\lambda_{h}=Z(\zeta)\left(C_{2} \dot{\zeta}+G_{2}+d_{2}(t)-B^{1} G_{r} K_{N} I\right) \\
v=L_{a} \frac{d I}{d t}+R_{a} I+K_{e} G_{r} T \dot{\zeta} .
\end{gathered}
$$

Until now we have brought the kinematics (2.3), dynamics (3.5), (3.6) and actuator dynamics (3.7) of the considered nonholonomic system from the generalized coordinate system $q \in R^{n}$ to feasible independent generalized velocities $\zeta \in R^{n-l-k}$ without violating the nonholonomic constraint (2.3). 


\section{Problem Statement}

Since the system is subjected to the nonholonomic constraint (2.3) and holonomic constraint (2.2), the states $q_{v}, q_{a}^{1}, q_{a}^{2}$ are not independent. By a proper partition of $q_{a}, q_{a}^{2}$ is uniquely determined by $\zeta=\left[\eta, q_{a}^{1}\right]^{T}$. Therefore, it is not necessary to consider the control of $q_{a}^{2}$.

Given a desired motion trajectory $\zeta^{d}(t)=\left[\eta^{d} q_{a}^{1^{d}}\right]^{T}$ and a desired constraint force $f_{d}(t)$, or, equivalently, a desired multiplier $\lambda_{h}(t)$, the trajectory and force tracking control is to determine a control law such that for any $(\zeta(0), \dot{\zeta}(0)) \in \Omega, \zeta, \dot{\zeta}, \lambda$ asymptotically converge to a manifold $\Omega_{d}$ specified as $\Omega$ where

$$
\Omega_{d}=\left\{\left(\zeta, \dot{\zeta}, \lambda_{h}\right) \mid \zeta=\zeta_{d}, \dot{\zeta}=\dot{\zeta}_{d}, \lambda=\lambda_{d}\right\}
$$

The controller design will consist of two stages: (i) a virtual adaptive control input $I^{d}$ is designed so that the subsystems (3.5) and (3.6) converge to the desired values, and (ii) the actual control input $v$ is designed in such a way that $I \rightarrow I^{d}$. In turn, this allows $\zeta-\zeta^{d}$ and $\lambda-\lambda^{d}$ to be stabilized to the origin.

Assumption 4.1. The desired reference trajectory $\zeta^{d}(t)$ is assumed to be bounded and uniformly continuous and has bounded and uniformly continuous derivatives up to the second order. The desired Lagrangian multiplier $\lambda_{d}(t)$ is also bounded and uniformly continuous.

\section{Robust Control Design}

\subsection{Kinematic and Dynamic Subsystems}

Let $e_{\zeta}=\zeta-\zeta^{d}, \dot{\zeta}_{r}=\dot{\zeta}^{d}-k_{\zeta} e_{\zeta}, r=\dot{e}_{\zeta}+k_{\zeta} e_{\zeta}$ with $k_{\zeta}>0, e_{\beta}=\lambda-\lambda^{d}$. A decoupled control scheme is introduced to control generalized position and constraint force separatively.

Consider the virtual control input $I$ is designed as

$$
I=K_{N}^{-1} G_{r}^{-1} B^{1^{-1}} \tau
$$

Let the control $u$ be as the form

$$
\begin{aligned}
& u=L^{+T} u_{a}-J^{1 T} u_{b}, \\
& u_{a}=B^{1} G_{r} K_{N a} I_{a}, \\
& u_{b}=B^{1} G_{r} K_{N b} I_{b},
\end{aligned}
$$

where $u_{a}, I_{a} \in R^{n-l-k}$ and $u_{b}, I_{b} \in R^{k}$ and $L^{+T}=\left(L^{T} L\right)^{-1} L^{T}$. Then, (2.13) and (2.14) can be changed to

$$
\begin{gathered}
M_{L}(\zeta) \ddot{\zeta}+C_{L}(\zeta, \dot{\zeta}) \dot{\zeta}+G_{L}+d_{L}(t)=B^{1} G_{r} K_{N a} I_{a} \\
Z(\zeta)\left(C^{1}(\zeta, \dot{\zeta}) \dot{\zeta}+G^{1}+d^{1}(t)-L^{+T} B^{1} G_{r} K_{N a} I_{a}\right)+B^{1} G_{r} K_{N b} I_{b}=\lambda_{h} .
\end{gathered}
$$


Consider the following control laws:

$$
\begin{gathered}
B^{1} G_{r} K_{N a} I_{a}^{d}=-K_{p} r-K_{i} \int r d t-\frac{r \Phi^{2}}{\Phi \gamma(\|r\|)+\delta}, \\
\Phi=C^{T} \Psi \\
B^{1} G_{r} K_{N b} I_{b}^{d}=\frac{x^{2}}{x+\delta}+\lambda_{h}^{d}-K_{f} e_{\lambda} \\
x=c_{1}\|Z(\zeta)\|\left\|L^{+T}\right\|\left\|\frac{d}{d t}\left[\dot{\zeta}^{d}\right]\right\|
\end{gathered}
$$

where $C=\left[\begin{array}{lllll}c_{1} & c_{2} & c_{3} & c_{4} & c_{5}\end{array}\right] ; \Psi=\left[\left\|(d / d t)\left[\dot{\zeta}_{r}\right]\right\|\left\|\dot{\zeta}_{r}\right\|\|\dot{\zeta}\|\left\|\dot{\zeta}_{r}\right\| 11\right]^{T} ; K_{p}, K_{i}, K_{f}$ are positive definite. $\gamma(\|r\|)$ can be defined as follows: if $\|r\| \leq \rho, \gamma(\|r\|)=\rho$, else $\gamma(\|r\|)=\|r\|, \rho$ is a small value, $\delta(t)$ is a time-varying positive function converging to zero as $t \rightarrow \infty$, such that $\int_{0}^{t} \delta(\omega) d \omega=a<\infty$. There are many choices for $\delta(t)$ that satisfies the condition.

\subsection{Control Design at the Actuator Level}

Till now, we have designed a virtual controller $I$ and $\zeta$ for kinematic and dynamic subsystems. $\zeta$ tending to $\zeta^{d}$ can be guaranteed, if the actual input control signal of the dynamic system $I$ be of the form $I^{d}$ which can be realized from the actuator dynamics by the design of the actual control input $v$. On the basis of the above statements we can conclude that if $v$ is designed in such a way that $I$ tends to $I^{d}$, then $\left(\zeta-\zeta^{d}\right) \rightarrow 0$ and $\left(\lambda-\lambda^{d}\right) \rightarrow 0$.

Defining $I=e_{I}+I^{d}$ and substituting $I$ and $\dot{\zeta}$ of (3.7) one gets

$$
L_{a} \dot{e}_{I}+R_{a} e_{I}+K_{e} G_{r} T \dot{e}_{\zeta}=-L_{a} \dot{I}^{d}-R_{a} I^{d}-K_{e} G_{r} T \dot{\zeta}^{d}+v
$$

The actuator parameters $K_{N}, L_{a}, R_{a}$, and $K_{e}$ are considered unknown for control design; however, there exist $L_{0}, R_{0}$, and $K_{e 0}$, such that

$$
\left\|L_{a}-L_{0}\right\| \leq \alpha_{1}, \quad\left\|R_{a}-R_{0}\right\| \leq \alpha_{2}, \quad\left\|K_{e}-K_{e 0}\right\| \leq \alpha_{3} .
$$

Consider the robust control law

$$
v=v_{0}-\sum_{i=1}^{3} \frac{e_{I} \mu_{i}^{2}}{\left\|e_{I}\right\| \mu_{i}+\delta}-K_{d} e_{I},
$$


where

$$
\begin{gathered}
v_{0}=L_{0} \dot{I}^{d}+R_{0} I^{d}+K_{e 0} G_{r} T \dot{\zeta}^{d}, \\
\mu_{1}=\alpha_{1}\left\|\left(\frac{d}{d t}\right) I^{d}\right\|, \\
\mu_{2}=\alpha_{2}\left\|I^{d}\right\|, \\
\mu_{3}=\alpha_{3}\left\|\left(\frac{d}{d t}\right) \zeta^{d}\right\| .
\end{gathered}
$$

\subsection{Stability Analysis for the System}

Theorem 5.1. Consider the mechanical system described by (2.1), (2.3), and (2.2); using the control law (5.5) and (5.7), the following hold for any $(q(0), \dot{q}(0)) \in \Omega_{n} \cap \Omega_{h}$ :

(i) $r$ and $e_{I}$ converge to a set containing the origin with the convergence rate as $t \rightarrow \infty$;

(ii) $e_{q}$ and $\dot{e}_{q}$ asymptotically converge to 0 as $t \rightarrow \infty$;

(iii) $e_{\lambda}$ and $\tau$ are bounded for all $t \geq 0$.

Proof. (i) By combing (3.5) with (5.5), the closed-loop system dynamics can be rewritten as

$$
M_{L} \dot{r}=B^{1} G_{r} K_{N a} I_{a}^{d}+B^{1} G_{r} K_{N a} e_{I}-\left(M_{L} \ddot{\zeta}_{r}+C_{L} \dot{\zeta}_{r}+G_{L}+d_{L}\right)-C_{L} r
$$

Substituting (5.5) into (5.13), the closed-loop dynamic equation is obtained:

$$
M_{L} \dot{r}=-K_{p} r-K_{i} \int r d t-\frac{r \Phi^{2}}{\Phi \gamma(\|r\|)+\delta}-\mu-C_{L} r+B^{1} G_{r} K_{N a} e_{I},
$$

where $\mu=M_{L} \ddot{\zeta}_{r}+C_{L} \dot{\zeta}_{r}+G_{L}+d_{L}$.

Consider the function

$$
\begin{aligned}
V & =V_{1}+V_{2}, \\
V_{1} & =\frac{1}{2} r^{T} M_{L} r+\frac{1}{2}\left(\int r d t\right)^{T} K_{i} \int r d t+e_{\zeta}^{T} k_{\zeta} K_{N a} K_{p} e_{\zeta}, \\
V_{2} & =\frac{1}{2} e_{I}^{T} K_{N a} L_{a} e_{I} .
\end{aligned}
$$

Then, differentiating $V_{1}$ with respect to time, we have

$$
\dot{V}_{1}=r^{T}\left(M_{L} \dot{r}+\frac{1}{2} \dot{M}_{L} r+K_{i} \int r d t\right)+2 e_{\zeta}^{T} k_{\zeta} K_{N a} K_{p} \dot{e}_{\zeta} .
$$


Journal of Applied Mathematics

From Property 1, we have $(1 / 2) \lambda_{\min }\left(M_{L}\right) r^{T} r \leq V \leq(1 / 2) \lambda_{\max }\left(M_{L}\right) r^{T} r$. By using Property 2, the time derivative of $V$ along the trajectory of (5.14) is

$$
\begin{aligned}
\dot{V}_{1} & =-r^{T} K_{p} r-r^{T} \mu-\frac{\|r\|^{2} \Phi^{2}}{\Phi \gamma(\|r\|)+\delta}+2 e_{\zeta}^{T} k_{\zeta} K_{N a} K_{p} \dot{e}_{\zeta}+r^{T} B^{1} G_{r} K_{N a} e_{I} \\
& \leq-r^{T} K_{p} r-\frac{\|r\|^{2} \Phi^{2}}{\Phi \gamma(\|r\|)+\delta}+\|r\| \Phi+2 e_{\zeta}^{T} k_{\zeta} K_{N a} K_{p} \dot{e}_{\zeta}+r^{T} B^{1} G_{r} K_{N a} e_{I} \\
& \leq-r^{T} K_{p} r-\frac{\|r\|^{2} \Phi^{2}-r(\|r\|) \Phi^{2}\|r\|-\|r\| \Phi \delta}{\Phi \gamma(\|r\|)+\delta}+2 e_{\zeta}^{T} k_{\zeta} K_{N a} K_{p} \dot{e}_{\zeta}+r^{T} B^{1} G_{r} K_{N a} e_{I},
\end{aligned}
$$

when $\|r\| \geq \rho$; therefore,

$$
\dot{V}_{1} \leq-r^{T} K_{p} r+\delta+2 e_{\zeta}^{T} k_{\zeta} K_{N a} K_{d} r-2 e_{\zeta}^{T} k_{\zeta} K_{N a} K_{p} k_{\zeta} e_{\zeta}+r^{T} B^{1} G_{r} K_{N a} e_{I} .
$$

Differentiating $V_{2}(t)$ with respect to time, using (3.7), one has

$$
\dot{V}_{2}=-e_{I}^{T} K_{N a}\left[L_{a} \dot{I}_{a}^{d}+R_{a} I_{a}^{d}+K_{e} G_{r} T \dot{\zeta}^{d}+R_{a} e_{I}+K_{e} G_{r} T \dot{e}_{\zeta}-v\right]
$$

Substituting $v$ in (5.19) by the control law (5.11), one has

$$
\begin{aligned}
\dot{V}_{2}= & -e_{I}^{T} K_{N a}\left(K_{d}+R_{a}\right) e_{I}-e_{I}^{T} K_{N a} K_{e} G_{r} T \dot{e}_{\zeta}-e_{I}^{T} K_{N a}\left(L_{a}-L_{0}\right) \dot{I}^{d} \\
& -e_{I}^{T} K_{N a}\left(R_{a}-R_{0}\right) I^{d}-e_{I}^{T} K_{N a}\left(K_{e}-K_{e 0}\right) G_{r} T \dot{\zeta}^{d}-e_{I}^{T} K_{N a} \sum_{i=1}^{3} \frac{\mu_{i}^{2} e_{I}}{\left\|e_{I}\right\| \mu_{i}+\delta} \\
\leq & -e_{I}^{T} K_{N a}\left(K_{d}+R_{a}\right) e_{I}-e_{I}^{T} K_{N a} K_{e} G_{r} T \dot{e}_{\zeta}+\alpha_{1} K_{N a}\left\|e_{I}\right\|\left\|\dot{I}^{d}\right\| \\
& +\alpha_{2} K_{N a}\left\|e_{I}\right\|\left\|I_{a}^{d}\right\|+\alpha_{3} K_{N a} G_{r} T\left\|e_{I}\right\|\left\|\zeta^{d}\right\|-K_{N a} \sum_{i=1}^{3} \frac{\left\|e_{I}\right\|^{2} \mu_{i}^{2}}{\left\|e_{I}\right\| \mu_{i}+\delta} \\
\leq & -e_{I}^{T} K_{N a}\left(K_{d}+R_{a}\right) e_{I}-\mathrm{e}_{I}^{T} K_{N a} K_{e} G_{r} T \dot{e}_{\zeta}+K_{N a} \sum_{i=1}^{3} \alpha_{i} \delta \\
= & -e_{I}^{T} K_{N a}\left(K_{d}+R_{a}\right) e_{I}-e_{I}^{T} K_{N} K_{e} G_{r} T r+e_{I}^{T} K_{N a} K_{e} G_{r} T k_{\zeta} e_{\zeta}+K_{N a} \delta \sum_{i=1}^{3} \alpha_{i} .
\end{aligned}
$$

Integrating (5.18) and (5.20), $\dot{V}$ can be expressed as

$$
\begin{aligned}
\dot{V} \leq & -r^{T} K_{p} r+\delta+2 e_{\zeta}^{T} k_{\zeta} K_{N a} K_{p} r-2 e_{\zeta}^{T} k_{\zeta} K_{N a} K_{p} k_{\zeta} e_{\zeta}+r^{T} B^{1} G_{r} K_{N a} e_{I} \\
& -e_{I}^{T} K_{N a}\left(K_{d}+R_{a}\right) e_{I}-e_{I}^{T} K_{N a} K_{e} G_{r} T r+e_{I}^{T} K_{N a} K_{e} G_{r} T k_{\zeta} e_{\zeta}+K_{N a} \delta \sum_{i=1}^{3} \alpha_{i} .
\end{aligned}
$$


We can obtain

$$
\dot{V} \leq-\left[\begin{array}{lll}
r^{T} & e_{\zeta} & e_{I}
\end{array}\right] Q\left[\begin{array}{ccc}
K_{N a} & 0 & 0 \\
0 & K_{N a} & 0 \\
0 & 0 & K_{N a}
\end{array}\right]\left[\begin{array}{c}
r \\
e_{\zeta} \\
e_{I}
\end{array}\right]
$$

where

$$
Q=\left[\begin{array}{ccc}
K_{p} & -K_{p} k_{\zeta} & \frac{1}{2} G_{r}\left(K_{e} T-B^{1}\right) \\
-k_{\zeta} K_{p} & 2 k_{\zeta} K_{p} T k_{\zeta} & -\frac{1}{2} K_{e} G_{r} T k_{\zeta} \\
\frac{1}{2} G_{r}\left(K_{e} T-B^{1}\right) & -\frac{1}{2} K_{e} G_{r} T k_{\zeta} & \left(K_{d}+R_{a}\right)
\end{array}\right] .
$$

The term $Q$ on the right-hand side (5.22) can always be negative definite by choosing suitable $K_{p}$ and $K_{d}$. Since $\left[K_{n a}\right]$ is positive definite, we only need to choose $K_{p}$ and $K_{d}$ such that $Q$ is positive definite. Therefore, $K_{d}$ and $K_{p}$ can always be chosen to satisfy

$$
\left(K_{d}+R\right)>K_{p}^{-1}\left[\frac{1}{2} G_{r}\left(K_{e} T-B^{1}\right)-\frac{1}{2} K_{e} G_{r} T k_{\zeta}\right]\left[\begin{array}{cc}
2 I & k_{\zeta}^{-1} \\
k_{\zeta}^{-1} & k_{\zeta}^{-1} T^{-1} k_{\zeta}^{-1}
\end{array}\right]\left[\begin{array}{c}
\frac{1}{2} G_{r}\left(K_{e} T-B^{1}\right) \\
-\frac{1}{2} K_{e} G_{r} T k_{\zeta}
\end{array}\right] .
$$

If $\|r\| \leq \rho$, it is easy to obtain $\dot{V} \leq 0 . r, e_{\zeta}$, and $e_{I}$ converge to a set containing the origin with $t \rightarrow \infty$.

(ii) $V$ is bounded, which implies that $r \in L_{\infty}^{n-k}$. From $r=\dot{e}_{\zeta}+k_{\zeta} e_{\zeta}$, it can be obtained that $e_{\zeta}, \dot{e}_{\zeta} \in L_{\infty}^{n-k}$. As we have established $e_{\zeta}, \dot{e}_{\zeta} \in L_{\infty}$, from Assumption 4.1, we conclude that $\zeta(t), \dot{\zeta}(t), \dot{\zeta}_{r}(t), \ddot{\zeta}_{r}(t) \in L_{\infty}^{n-k}$ and $\dot{q} \in L_{\infty}^{n}$.

Therefore, all the signals on the right hand side of (5.14) are bounded, and we can conclude that $\dot{r}$ and therefore $\ddot{\zeta}$ are bounded. Thus, $r \rightarrow 0$ as $t \rightarrow \infty$ can be obtained. Consequently, we have $e_{\zeta} \rightarrow 0, \dot{e}_{\zeta} \rightarrow 0$ as $t \rightarrow \infty$. It follows that $e_{q}, \dot{e}_{q} \rightarrow 0$ as $t \rightarrow \infty$.

(iii) Substituting the control (5.5) and (5.7) into the reduced order dynamic system model (5.4) yields

$$
\begin{aligned}
\left(1+K_{f}\right) e_{\lambda} & =Z(\zeta)\left(C^{1}(\zeta, \dot{\zeta}) \dot{\zeta}+G^{1}+d^{1}(t)-L^{+T} G_{r} K_{N a} I_{a}\right)+B^{1} G_{r} K_{N b} I_{b}^{d}+B^{1} G_{r} K_{N b} e_{I} \\
& =-Z(\zeta) L^{+T} M_{L}(\zeta)(\ddot{\zeta})+\frac{x^{2}}{x+\delta}+B^{1} G_{r} K_{N b} e_{I}
\end{aligned}
$$

Since $\dot{\zeta}=0$ when $I \in R^{k}$, (3.7) could be changed as

$$
L_{a} \frac{d I_{b}}{d t}+R_{a} I_{b}=v_{b}
$$




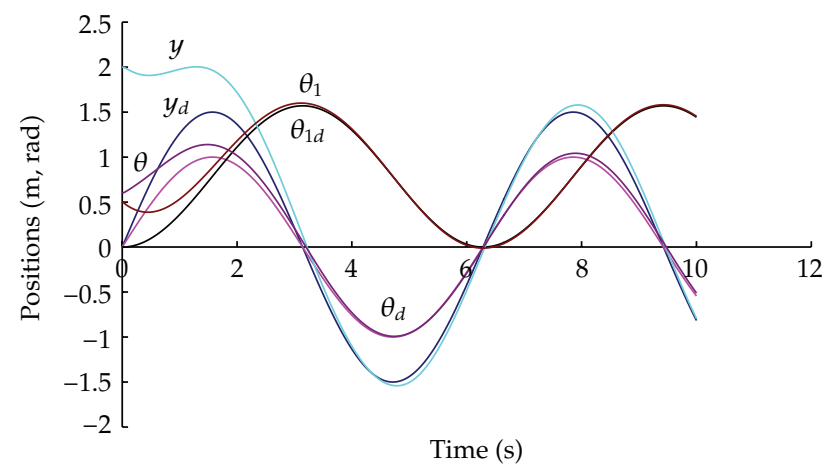

Figure 2: The positions of the joints.

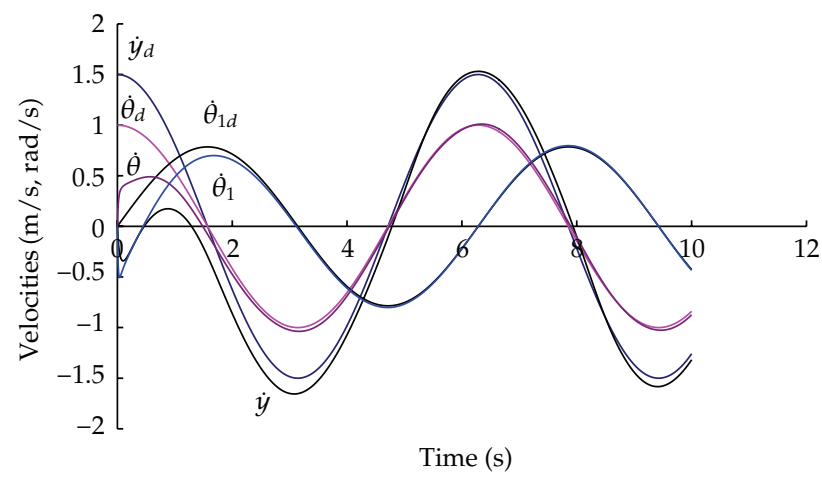

Figure 3: The velocities of the joints.

Therefore, $r=0$ and $e_{\zeta}=0$ in the force space; (5.20) could be changed as

$$
\dot{V}_{2}=-e_{I}^{T} K_{N b}\left(K_{d}+R\right) e_{I}+K_{N b} \delta \sum_{i=1}^{3} \alpha_{i}
$$

Since $K_{N b}$ is bounded, $\dot{V}<0$, we can obtain $e_{I} \rightarrow 0$ as $t \rightarrow \infty$. The proof is completed by noticing that $\ddot{\zeta}, Z(q), K_{N b}$ and $e_{I}$ are bounded. Moreover, $\zeta \rightarrow \zeta^{d}$, and $-Z(\zeta) L^{+T} M_{L}(\zeta)\left(\ddot{\zeta}^{d}\right)+$ $x^{2} /(x+\delta) \leq \delta, e_{I} \rightarrow 0$, the right-hand side terms of (5.25), tend uniformly asymptotically to zero; then it follows that $e_{\lambda} \rightarrow 0$, then $f(t) \rightarrow f_{d}(t)$.

Since $r, \zeta, \dot{\zeta}, \zeta_{r}, \dot{\zeta}_{r}, \ddot{\zeta}_{r}, e_{\lambda}$ and $e_{I}$ are all bounded, it is easy to conclude that $\tau$ is bounded from (5.2).

\section{Simulations}

To verify the effectiveness of the proposed control algorithm, let us consider a 2-DOF manipulator mounted on two-wheels-driven mobile base [23] shown in Figure 1. The mobile manipulator is subjected to the following constraints: $\dot{x} \cos \theta+\dot{y} \sin \theta=0$. Using Lagrangian 
approach, we can obtain the standard form with $q_{v}=[x, y, \theta]^{T}, q_{a}=\left[\theta_{1}, \theta_{2}\right]^{T}, q=\left[q_{v}, q_{a}\right]^{T}$, and $A_{v}=[\cos \theta, \sin \theta, 0]^{T}$ :

$$
\begin{aligned}
& M_{v}=\left[\begin{array}{ccc}
m_{p 12}+\frac{2 I_{w} \sin ^{2} \theta}{r^{2}} & -\frac{2 I_{w}}{r^{2}} \sin \theta \cos \theta & -m_{12} d \sin \theta \\
-\frac{2 I_{w}}{r^{2}} \sin \theta \cos \theta & m_{p 12}+\frac{2 I_{w} \cos ^{2} \theta}{r^{2}} & m_{12} d \cos \theta \\
-m_{12} d \sin \theta & m_{12} d \cos \theta & M_{11}^{1}
\end{array}\right], \\
& M_{11}^{1}=I_{p}+I_{12}+m_{12} d^{2}+\frac{2 I_{w} L^{2}}{r^{2}}, \quad M_{a}=\operatorname{diag}\left[I_{12}, I_{2}\right] \\
& M_{v a}=\left[\begin{array}{ll}
0.0 & 0.0 \\
0.0 & 0.0 \\
I_{12} & 0.0
\end{array}\right], \\
& B=\left[\begin{array}{cccc}
\frac{\sin \theta}{r} & -\frac{\sin \theta}{r} & 0.0 & 0.0 \\
-\frac{\cos \theta}{r} & \frac{\cos \theta}{r} & 0.0 & 0.0 \\
-\frac{l}{r} & \frac{l}{r} & 0.0 & 0.0 \\
0.0 & 0.0 & 1.0 & 0.0 \\
0.0 & 0.0 & 0.0 & 1.0
\end{array}\right], \\
& C_{v}=\left[\begin{array}{cccc}
\frac{2 I_{w}}{r^{2}} \dot{\theta} \sin \theta \cos \theta & \frac{2 I_{w}}{r^{2}} \dot{\theta} \sin ^{2} \theta & -m_{12} d \dot{\theta} \cos \theta & 0.0 \\
-\frac{2 I_{w}}{r^{2}} \dot{\theta} \cos ^{2} \theta & \frac{2 I_{w}}{r^{2}} \dot{\theta} \sin \theta \cos \theta & m_{12} d \dot{\theta} \cos \theta & 0.0 \\
0.0 & 0.0 & 0.0 & 0.0
\end{array}\right] \text {, } \\
& C_{v a}=0.0, \quad C_{a}=0.0, \quad G_{v}=[0.0,0.0,0.0]^{T}, \quad G_{a}=\left[0.0, m_{2} g l_{2} \sin \theta_{2}\right]^{T} \text {, } \\
& H=\left[\begin{array}{cccc}
-\tan \theta & 0.0 & 0.0 & 0.0 \\
1.0 & 0.0 & 0.0 & 0.0 \\
0.0 & 1.0 & 0.0 & 0.0 \\
0.0 & 0.0 & 1.0 & 0.0 \\
0.0 & 0.0 & 0.0 & 1.0
\end{array}\right] \\
& \tau_{v}=\left[\tau_{l}, \tau_{r}\right]^{T}, \quad \tau_{a}=\left[\tau_{1}, \tau_{2}\right]^{T}, \\
& m_{p 12}=m_{p}+m_{12}, \quad m_{12}=m_{1}+m_{2}, \quad I_{12}=I_{1}+I_{2} \text {. }
\end{aligned}
$$

Let the desired trajectory $q_{d}=\left[x_{d}, y_{d}, \theta_{d}, \theta_{1 d}, \theta_{2 d}\right]^{T}$ and the end effector be subject to the geometric constraint $\Phi=l_{1}+l_{2} \sin \left(\theta_{2}\right)=0$, and $y_{d}=1.5 \sin (t), \theta_{d}=1.0 \sin (t), \theta_{1 d}=$ $\pi / 4(1-\cos (t)), \lambda_{d}=10.0 N$.

The trajectory and force tracking control problem is to design control law $\tau$ such that (4.1) holds and all internal signals are bounded. 


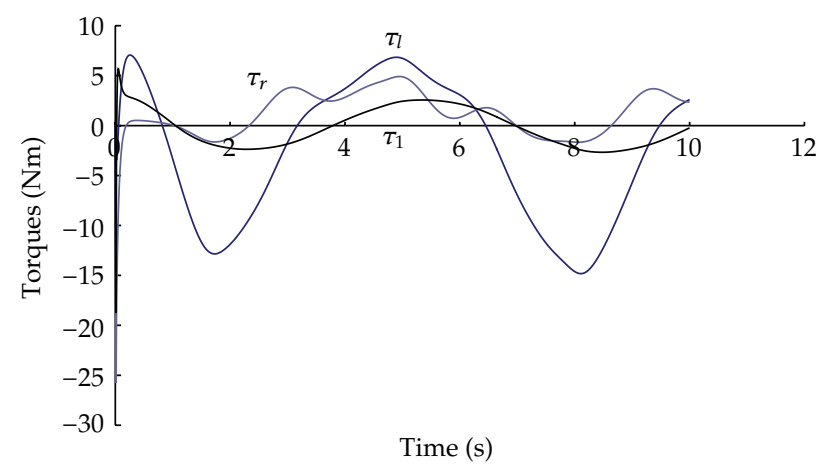

Figure 4: The torques of the joints.

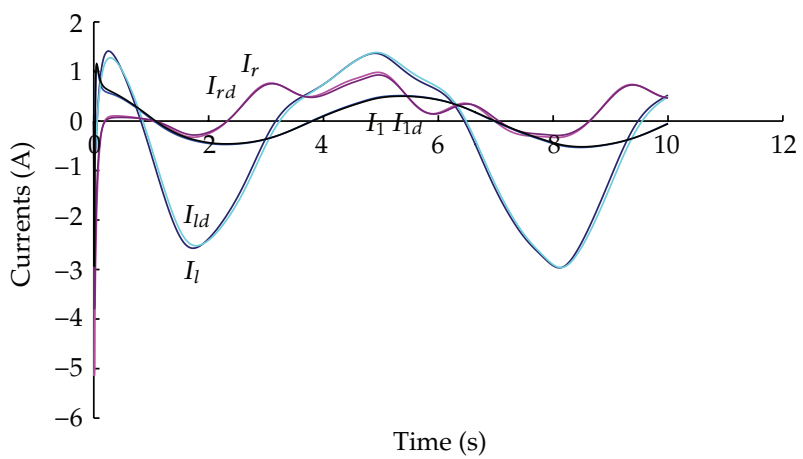

Figure 5: Tracking the desired currents.

In the simulation, we assume the parameter $m_{p}=m_{1}=m_{2}=1.0, I_{w}=I_{p}=1.0,2 I_{1}=$ $I_{2}=1.0, I=0.5, d=L=R=1.0,2 l_{1}=1.0,2 l_{2}=0.6, q(0)=[0,2.0,0.6,0.5]^{T}, \dot{q}(0)=$ $[0.0,0.0,0.0,0.0]^{T}, K_{N}=\operatorname{diag}[0.01], G_{r}=\operatorname{diag}[100], L_{a}=[0.005,0.005,0.005,0.005]^{T}, R_{a}=$ $[2.5,2.5,2.5,2.5]^{T}$, and $K_{e}=[0.02,0.02,0.02,0.02]^{T}$. The disturbance on the mobile base is set $0.1 \sin (t)$ and $0.1 \cos (t)$. By Theorem 5.1, the control gains are selected as $K_{p}=$ $\operatorname{diag}[1.0,1.0,1.0], k_{\zeta}=\operatorname{diag}[1.0,1.0,1.0], K_{i}=0.0$ and $K_{f}=0.995, C=[8.0,8.0,8.0,8.0,8.0]^{T}$, $K_{N}=0.1, K_{d}=\operatorname{diag}[10,10,10,10], \alpha_{1}=0.008, \alpha_{2}=4.0, \alpha_{3}=0.03$. The disturbance on the mobile base is set $0.1 \sin (t)$ and $0.1 \cos (t)$. The simulation results for motion/force are shown in Figures 2, 3, 4, 5, 6, 7, 8, and 9. The desired currents tracking and input voltages on the motors are shown in Figures 5, 6, 8, and 9. The simulation results show that the trajectory and force tracking errors asymptotically tend to zero, which validate the effectiveness of the control law in Theorem 5.1.

\section{Conclusion}

In this paper, effective robust control strategies have been presented systematically to control the holonomic constrained nonholonomic mobile manipulator in the presence of uncertainties and disturbances, and actuator dynamics is considered in the robust control. All control strategies have been designed to drive the system motion converge to the desired 


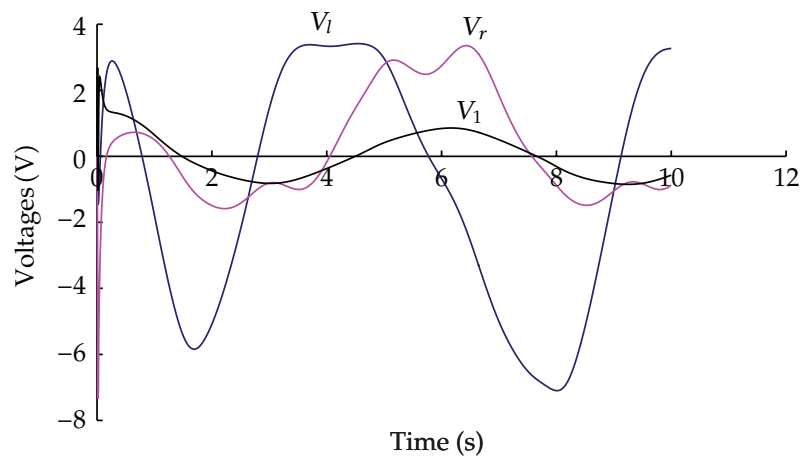

Figure 6: The input voltages.

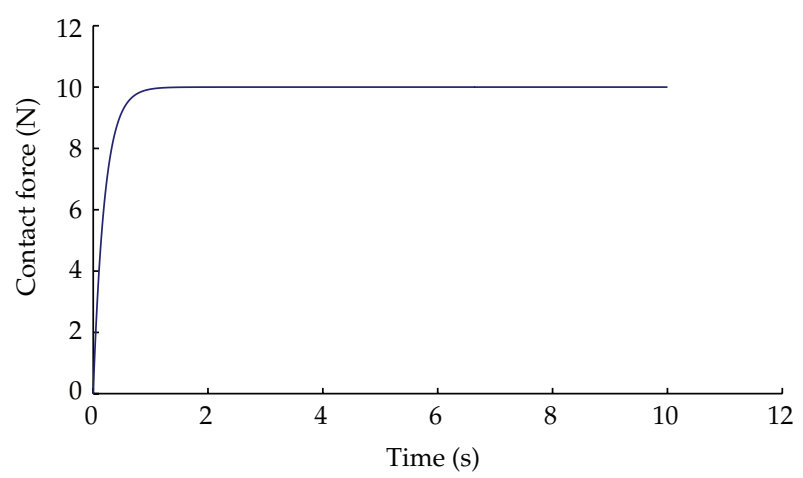

Figure 7: The constraint force.

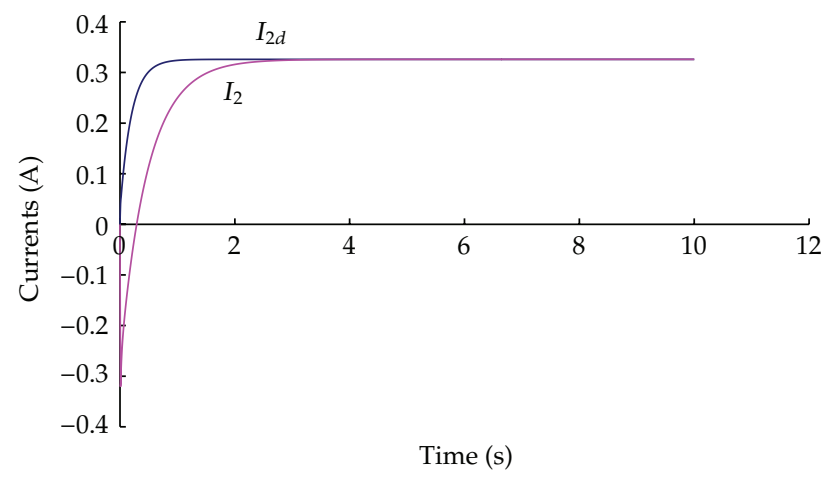

Figure 8: Tracking the desired current of the joint 2.

manifold and at the same time guarantee the boundedness of the constrained force. The proposed controls are nonregressor based and require no information on the system dynamics. Simulation studies have verified the effectiveness of the proposed controller. 


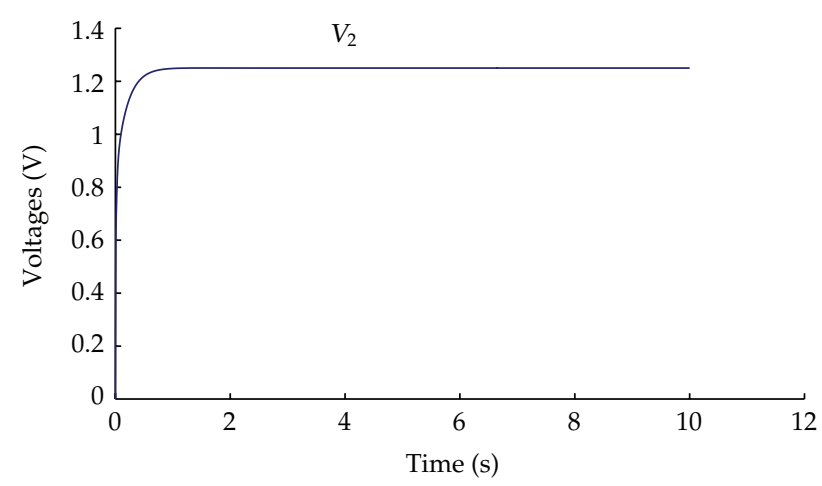

Figure 9: The input voltage of the joint 2.

\section{Acknowledgment}

The authors are thankful to the Ministry of Science and Technology of China as the paper is partially sponsored by the National High-Technology Research and Development Program of China (863 Program) (no. 2009AA012201).

\section{References}

[1] Z. Li, P. Y. Tao, S. S. Ge, M. Adams, and W. S. Wijesoma, "Robust adaptive control of cooperating mobile manipulators with relative motion," IEEE Transactions on Systems, Man, and Cybernetics, Part B, vol. 39, no. 1, pp. 103-116, 2009.

[2] Z. Li, S. S. Ge, and A. Ming, "Adaptive robust motion/force control of holonomic-constrained nonholonomic mobile manipulators," IEEE Transactions on Systems, Man, and Cybernetics, Part B, vol. 37, no. 3, pp. 607-616, 2007.

[3] Z. Li, C. Yang, J. Luo, Z. Wang, and A. Ming, "Robust motion/force control of nonholonomic mobile manipulators using hybrid joints," Advanced Robotics, vol. 21, no. 11, pp. 1231-1252, 2007.

[4] Z. Li, A. Ming, N. Xi, J. Gu, and M. Shimojo, "Development of hybrid joints for the compliant arm of human-symbiotic mobile manipulator," International Journal of Robotics and Automation, vol. 20, no. 4, pp. 260-270, 2005.

[5] V. A. Pavlov and A. V. Timofeyev, "Construction and stabilization of programmed movements of a mobile robot-manipulator," Engineering Cybernetics, vol. 14, no. 6, pp. 70-79, 1976.

[6] J. H. Chung and S. A. Velinsky, "Modeling and control of a mobile manipulator," Robotica, vol. 16, no. 6, pp. 607-613, 1998.

[7] K. Watanabe, K. Sato, K. Izumi, and Y. Kunitake, "Analysis and control for an omnidirectional mobile manipulator," Journal of Intelligent and Robotic Systems, vol. 27, no. 1-2, pp. 3-20, 2000.

[8] Z. Li, C. Yang, and N. Ding, "Robust adaptive motion control for remotely operated vehicles with velocity constraints," International Journal of Control, Automation, and System, vol. 10, no. 2, pp. 421429, 2012.

[9] Y. Kang, Z. Li, W. Shang, and H. Xi, "Control design for tele-operation system with time-varying and stochastic communication delays," International Journal of Innovative Computing, Information and Control, vol. 8, no. 1, pp. 61-74, 2012.

[10] Y. Kang, Z. Li, W. Shang, and H. Xi, "Motion synchronisation of bilateral teleoperation systems with mode-dependent time-varying communication delays," IET Control Theory E Applications, vol. 4, no. 10, pp. 2129-2140, 2010.

[11] Z. Li, X. Cao, and N. Ding, "Adaptive fuzzy control for synchronization of nonlinear teleoperators with stochastic time-varying communication delays," IEEE Transactions on Fuzzy Systems, vol. 19, no. 4, pp. 745-757, 2011.

[12] Z. Li and W. Chen, "Adaptive neural-fuzzy control of uncertain constrained multiple coordinated nonholonomic mobile manipulators," Engineering Applications of Artificial Intelligence, vol. 21, no. 7, pp. 985-1000, 2008. 
[13] Z. Li, S. S. Ge, and Z. Wang, "Robust adaptive control of coordinated multiple mobile manipulators," Mechatronics, vol. 18, no. 5-6, pp. 239-250, 2008.

[14] Y. Yamamoto and X. Yun, "Effect of the dynamic interaction on coordinated control of mobile manipulators," IEEE Transactions on Robotics and Automation, vol. 12, no. 5, pp. 816-824, 1996.

[15] Z. Li, W. Chen, and J. Luo, "Adaptive compliant force-motion control of coordinated non-holonomic mobile manipulators interacting with unknown non-rigid environments," Neurocomputing, vol. 71, no. 7-9, pp. 1330-1344, 2008.

[16] Z. Li, J. Gu, A. Ming, C. Xu, and M. Shimojo, "Intelligent compliant force/motion control of nonholonomic mobile manipulator working on the nonrigid surface," Neural Computing and Applications, vol. 15, no. 3-4, pp. 204-216, 2006.

[17] Y. Yamamoto and X. Yun, "Coordinating locomotion and manipulation of a mobile manipulator," IEEE Transactions on Automatic Control, vol. 39, no. 6, pp. 1326-1332, 1994.

[18] O. Khatib, "Mobile manipulation: the robotic assistant," Robotics and Autonomous Systems, vol. 26, no. 2-3, pp. 175-183, 1999.

[19] B. Bayle, J. Y. Fourquet, and M. Renaud, "Manipulability of wheeled mobile manipulators: application to motion generation," International Journal of Robotics Research, vol. 22, no. 7-8, pp. 565-581, 2003.

[20] J. Tan, N. Xi, and Y. Wang, "Integrated task planning and control for mobile manipulators," International Journal of Robotics Research, vol. 22, no. 5, pp. 337-354, 2003.

[21] S. Lin and A. A. Goldenberg, "Neural-network control of mobile manipulators," IEEE Transactions on Neural Networks, vol. 12, no. 5, pp. 1121-1133, 2001.

[22] Z. Li, C. Yang, and J. Gu, "Neuro-adaptive compliant force/motion control of uncertain constrained wheeled mobile manipulators," International Journal of Robotics and Automation, vol. 22, no. 3, pp. 206214, 2007.

[23] W. Dong, “On trajectory and force tracking control of constrained mobile manipulators with parameter uncertainty," Automatica, vol. 38, no. 9, pp. 1475-1484, 2002.

[24] Z. Li, S. S. Ge, M. Adams, and W. S. Wijesoma, "Robust adaptive control of uncertain force/motion constrained nonholonomic mobile manipulators," Automatica, vol. 44, no. 3, pp. 776-784, 2008.

[25] M. C. Good, L. M. Sweet, and K. L. Strobel, "Dynamic models for control system design of integrated robot and drive systems," Journal of Dynamic Systems, Measurement and Control, vol. 107, no. 1, pp. 53-59, 1985.

[26] Z. Li, S. S. Ge, M. Adams, and W. S. Wijesoma, "Adaptive robust output-feedback motion/force control of electrically driven nonholonomic mobile manipulators," IEEE Transactions on Control Systems Technology, vol. 16, no. 6, pp. 1308-1315, 2008.

[27] Z. Li, J. Li, and Y. Kang, "Adaptive robust coordinated control of multiple mobile manipulators interacting with rigid environments," Automatica, vol. 46, no. 12, pp. 2028-2034, 2010.

[28] J. H. Yang, "Adaptive robust tracking control for compliant-join mechanical arms with motor dynamics," in Proceedings of the 38th IEEE Conference on Decision \& Control, pp. 3394-3399, December 1999.

[29] R. Colbaugh and K. Glass, "Adaptive regulation of rigid-link electrically-driven manipulators," in Proceedings of the IEEE International Conference on Robotics \& Automation. Part 1, pp. 293-299, May 1995.

[30] C. Y. Su and Y. Stepanenko, "Hybrid adaptive/robust motion control of rigid-link electrically-driven robot manipulators," IEEE Transactions on Robotics E Automation, vol. 11, no. 3, pp. 426-432, 1995.

[31] Z. P. Wang, S. S. Ge, and T. H. Lee, "Robust motion/force control of uncertain holonomic/nonholonomic mechanical systems," IEEE/ASME Transactions on Mechatronics, vol. 9, no. 1, pp. 118-123, 2004.

[32] S. S. Ge, J. Wang, T. H. Lee, and G. Y. Zhou, "Adaptive robust stabilization of dynamic nonholonomic chained systems," Journal of Robotic Systems, vol. 18, no. 3, pp. 119-133, 2001.

[33] S. S. Ge, Z. Wang, and T. H. Lee, "Adaptive stabilization of uncertain nonholonomic systems by state and output feedback," Automatica, vol. 39, no. 8, pp. 1451-1460, 2003.

[34] W. Dong, W. L. Xu, and W. Huo, "Trajectory tracking control of dynamic non-holonomic systems with unknown dynamics," International Journal of Robust and Nonlinear Control, vol. 9, no. 13, pp. 905-922, 1999.

[35] Z. Li, Y. Yang, and J. Li, "Adaptive motion/force control of mobile under-actuated manipulators with dynamics uncertainties by dynamic coupling and output feedback," IEEE Transactions on Control Systems Technology, vol. 18, no. 5, pp. 1068-1079, 2010.

[36] Z. Li and C. Xu, "Adaptive fuzzy logic control of dynamic balance and motion for wheeled inverted pendulums," Fuzzy Sets and Systems, vol. 160, no. 12, pp. 1787-1803, 2009.

[37] Z. Li and J. Luo, "Adaptive robust dynamic balance and motion controls of mobile wheeled inverted pendulums," IEEE Transactions on Control Systems Technology, vol. 17, no. 1, pp. 233-241, 2009. 
[38] Y. Kang, Z. Li, Y. Dong, and H. Xi, "Markovian-based fault-tolerant control for wheeled mobile manipulators," IEEE Transactions on Control Systems Technology, vol. 20, no. 1, pp. 266-276, 2012.

[39] Z. Li, "Adaptive fuzzy output feedback motion/force control for wheeled inverted pendulums," IET Control Theory \& Applications, vol. 5, no. 10, pp. 1176-1188, 2011.

[40] Z. Li and Y. Kang, "Dynamic coupling switching control incorporating support vector machines for wheeled mobile manipulators with hybrid joints," Automatica, vol. 46, no. 5, pp. 832-842, 2010.

[41] Z. Li and Y. Zhang, "Robust adaptive motion/force control for wheeled inverted pendulums," Automatica, vol. 46, no. 8, pp. 1346-1353, 2010.

[42] Z. Li, Y. Zhang, and Y. Yang, "Support vector machine optimal control for mobile wheeled inverted pendulums with unmodelled dynamics," Neurocomputing, vol. 73, no. 13-15, pp. 2773-2782, 2010.

[43] Z. Li, J. Zhang, and Y. Yang, "Motion control of mobile under-actuated manipulators by implicit function using support vector machines," IET Control Theory \& Applications, vol. 4, no. 11, pp. 23562368, 2010.

[44] C. Y. Su and Y. Stepanenko, "Robust motion/force control of mechanical systems with classical nonholonomic constraints," IEEE Transactions on Automatic Control, vol. 39, no. 3, pp. 609-614, 1994.

[45] N. H. McClamroch and D. Wang, "Feedback stabilization and tracking of constrained robots," IEEE Transactions on Automatic Control, vol. 33, no. 5, pp. 419-426, 1988.

[46] S. S. Ge, Z. Li, and H. Yang, "Data driven adaptive predictive control for holonomic constrained under-actuated biped robots," IEEE Transactions on Control Systems Technology, vol. 20, no. 3, pp. 787795, 2012.

[47] C. M. Anupoju, C. Y. Su, and M. Oya, "Adaptive motio tracking control of uncertainonholonomic mechanical systems including actuator dynamics," IEE Proceedings Control Theory E Applications, vol. 152 , no. 5, pp. 575-580, 2005. 


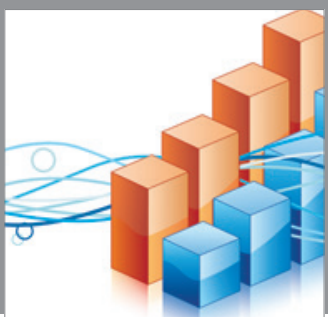

Advances in

Operations Research

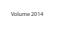

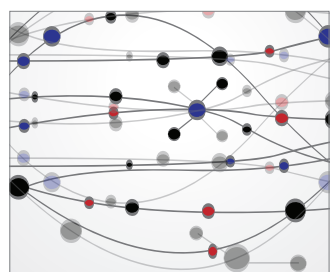

\section{The Scientific} World Journal
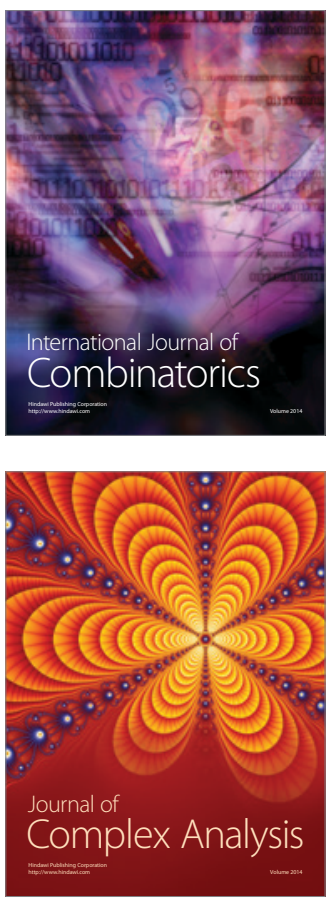

International Journal of

Mathematics and

Mathematical

Sciences
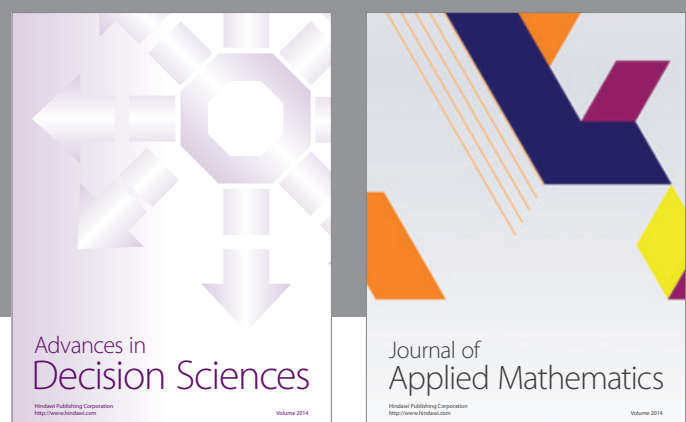

Journal of

Applied Mathematics
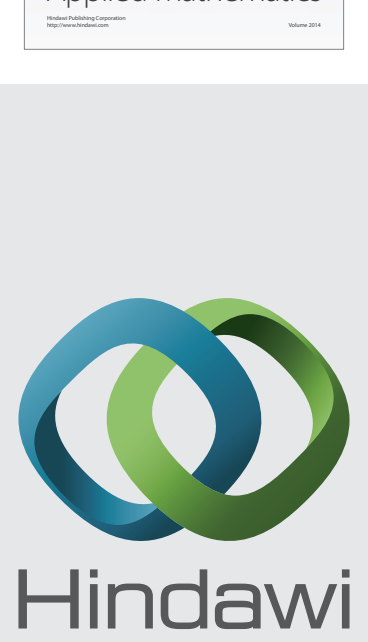

Submit your manuscripts at http://www.hindawi.com
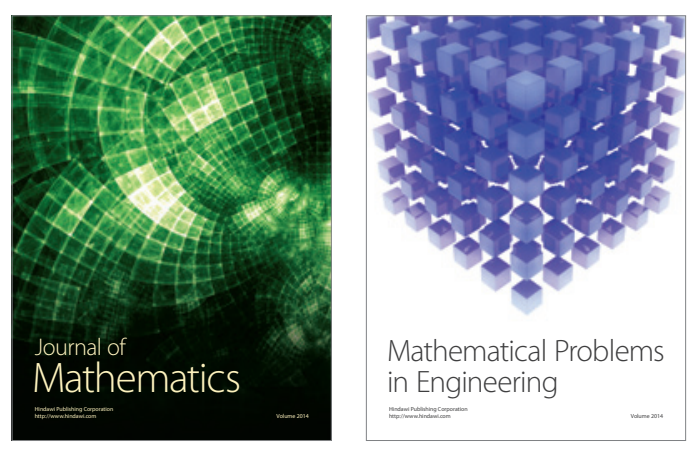

Mathematical Problems in Engineering
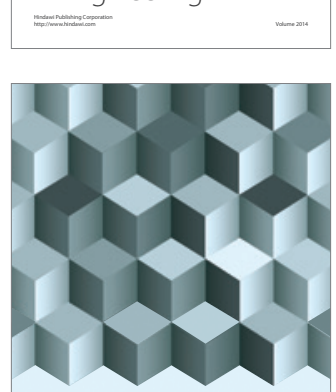

Journal of

Function Spaces
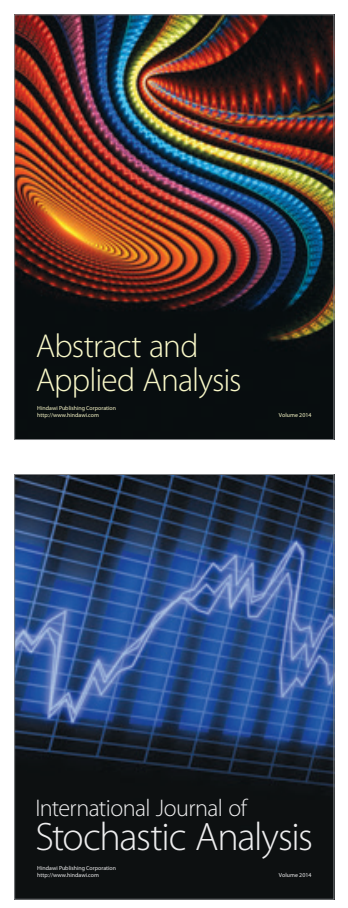

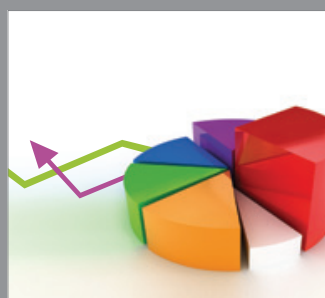

ournal of

Probability and Statistics

Promensencen
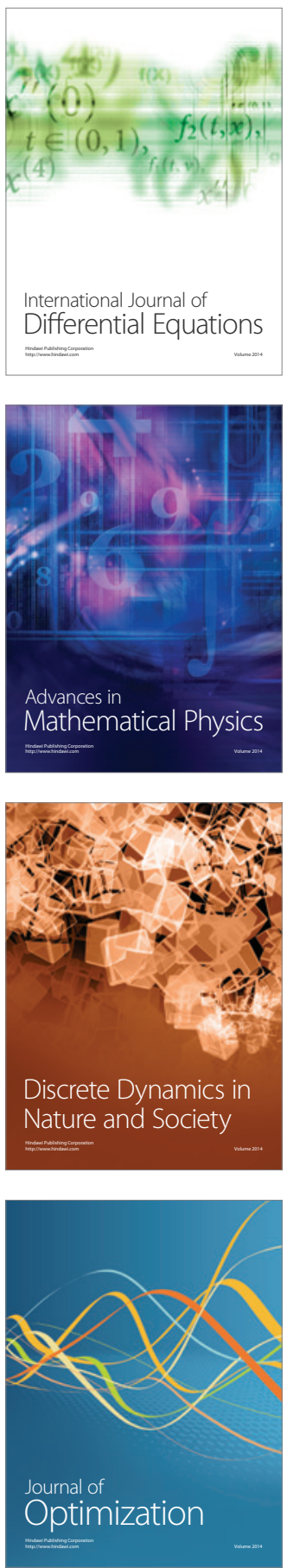